\title{
Operational experience with the Silicon Strip Tracker at the CMS experiment
}

\author{
Ivan Shvetsov*i \\ Karlsruhe Institute of Technology \\ E-mail: ivan.shvetsovecern.ch
}

\begin{abstract}
The CMS Silicon Strip Tracker with its more than 15000 silicon modules and $200 \mathrm{~m}^{2}$ of active silicon area is in its tenth year of operation at the LHC. We present the performance of the detector in the LHC Run 2 data taking. Results for signal-to-noise, hit efficiency and single hit resolution will be presented. We review the behavior of the system when running at beyond-design instantaneous luminosity and describe challenges observed under these conditions. The evolution of detector parameters under the influence of radiation damage will be presented and compared to simulations.
\end{abstract}

The 27th International Workshop on Vertex Detectors - VERTEX2018

22-26 October 2018

MGM Beach Resorts, Muttukadu, Chennai, India

\footnotetext{
* Speaker.

${ }^{\dagger}$ for the CMS collaboration
} 


\section{Introduction}

The CMS Silicon Strip Tracker (SST) is located in the centre of the CMS experiment. Together with the pixel detector it provides measurements of charged particle trajectories up to a pseudorapidity $|\eta|<2.5$. The layout of the CMS SST is shown in Fig.1. The SST has 9.3 million strips

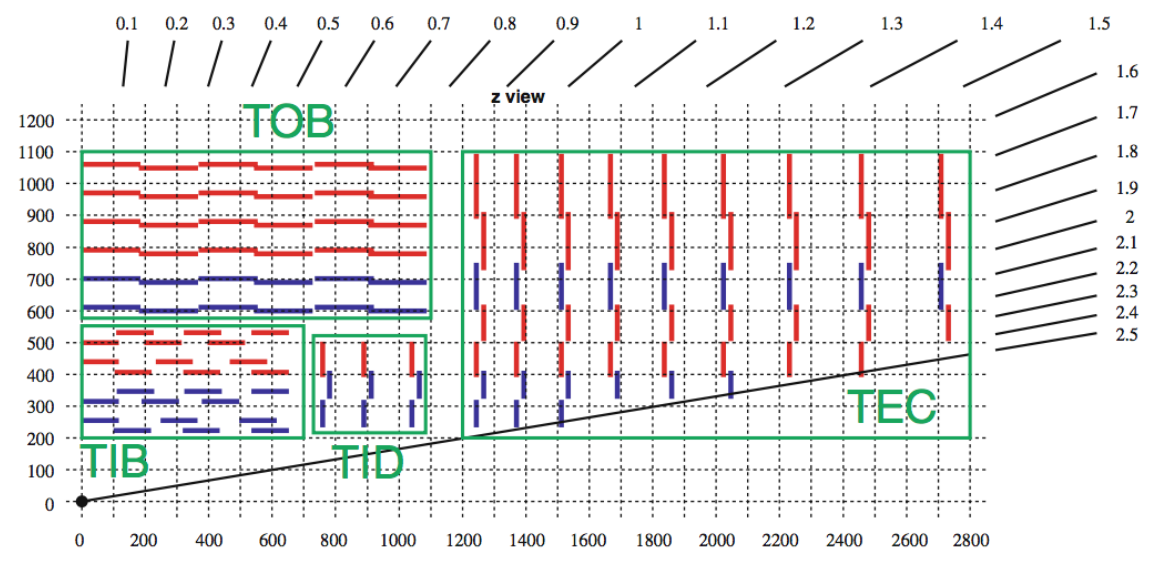

Figure 1: The layout of the CMS Silicon strip tracker [1].

and $198 \mathrm{~m}^{2}$ of active silicon area distributed over 15148 modules. Single-sided p-on-n micro-strip sensors are used. The detector is $5 \mathrm{~m}$ long and has a diameter of $2.5 \mathrm{~m}$. It has 10 layers in the barrel region with 4 layers in the Tracker Inner Barrel (TIB) and 6 layers in the Tracker Outer Barrel (TOB). The TIB is supplemented with 3 Tracker Inner Disks (TID) at each end. In the forward region the detector consists of Tracker End-Caps (TEC) at each end as shown in Fig. 1. Each TID is composed of 3 rings and each TEC is composed of up to 7 rings.

In TIB, TID and in rings 1-4 of TECs sensors with thickness of $320 \mu \mathrm{m}$ are used while in TOB and in rings 5-7 of TECs $500 \mu \mathrm{m}$ sensors are used. The geometry of modules used is summarized in Fig.2. In 4 layers in the barrel and in 3 rings in the end-caps so-called stereo modules are used (Fig. 1). These modules have a second module mounted back-to-back with a stereo angle of $100 \mathrm{mrad}$. They provide measurements of 3D coordinates ( $r$ in the disks and $z$ in the barrel).

Analog signals from sensors are processed by APV25 chips [4] and then converted to optical signals on analog-opto-hybrids $(\mathrm{AOH})$ and transmitted to front-end drivers (FED, located in the service cavern outside the radiation zone) via optical fibers. Pedestal and common mode subtraction as well as cluster finding are performed in the FEDs. Clock and trigger information is distributed by front-end controllers (FEC) to Communication and Control Units (CCU) grouped in token ring networks (control rings). The SST has 1944 power groups. Each power group has 2 low voltage channels with $2.5 \mathrm{~V}$ and $1.25 \mathrm{~V}$ regulators and 2 high voltage channels, which can be regulated up to $600 \mathrm{~V}$ [3]. The detector is cooled with $\mathrm{C}_{6} \mathrm{~F}_{14}$ monophase coolant by two cooling plants.

\section{Run 2 experience}

\subsection{Detector status}

The fraction of active readout channels in the detector is $96.2 \%$, which was largely stable in 


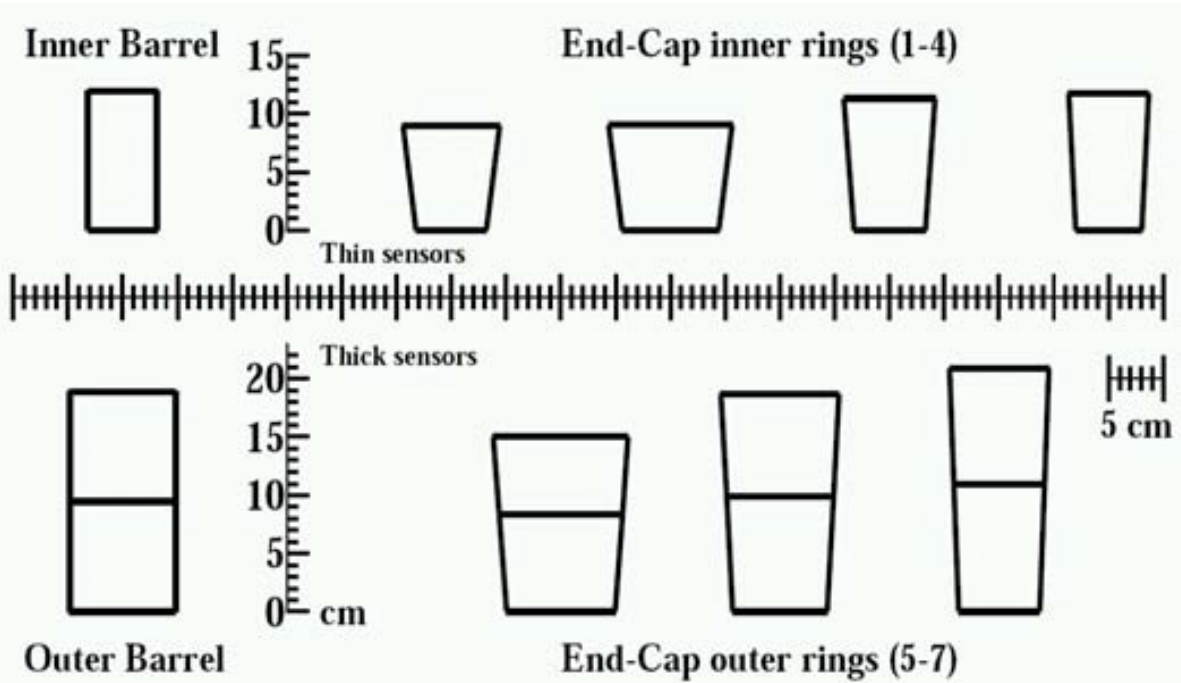

Figure 2: Geometry of sensors used in the CMS Strip Tracker [3].

the period of operation from spring 2015 till the end of 2018. Components excluded from datataking include failing control rings, problems in LV or HV distribution, and individually switched off modules.

\subsection{Detector performance}

The performance of the Strip Tracker is monitored continuously. One of the most important characteristics of the detector is the signal to noise ratio $(\mathrm{S} / \mathrm{N})$ of hits. The signal-to-noise distributions for TIB and TOB are shown in Fig.3. Each distribution can be fitted with a Landau convoluted with a normal distribution. The most probable value (MPV) of this distribution is taken as estimation of the $\mathrm{S} / \mathrm{N}$ for a given part of the SST. Measured values are shown in Tab. 1.
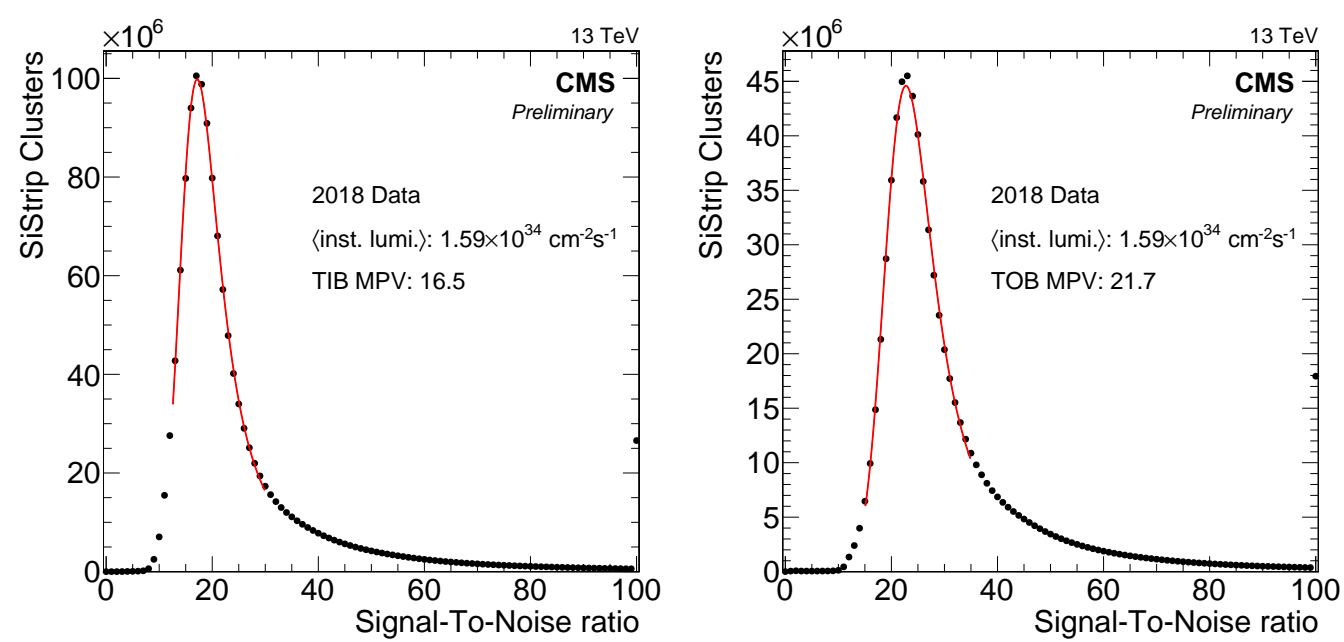

Figure 3: Signal to noise distribution for hits on reconstructed particle tracks for TIB (left) and TOB (right). 


\begin{tabular}{|c|c|}
\hline Sensor type & S/N[MPV] \\
\hline TIB & 16.5 \\
\hline TID & 16.0 \\
\hline TOB & 21.7 \\
\hline TEC (thin) & 16.9 \\
\hline TEC (thick) & 21.8 \\
\hline
\end{tabular}

Table 1: Most probable value for S/N distribution for different parts of the Silicon Strip Tracker measured with data recorded in 2018. For TECs values for thin $(320 \mu \mathrm{m})$ and thick $(500 \mu \mathrm{m})$ modules are given.

The evolution of the $\mathrm{S} / \mathrm{N}$ with integrated luminosity is shown in Fig.4. As expected from irradiation studies [7] the S/N drops with integrated fluence. Furthermore, the curve follows the expected trend. Based on the data collected so far, we estimate that $\mathrm{S} / \mathrm{N}$ of the Strip Tracker will be sufficient for high quality physics data taking up to the end of its operational lifetime $\left(\sim 500 \mathrm{fb}^{-1}\right)$.

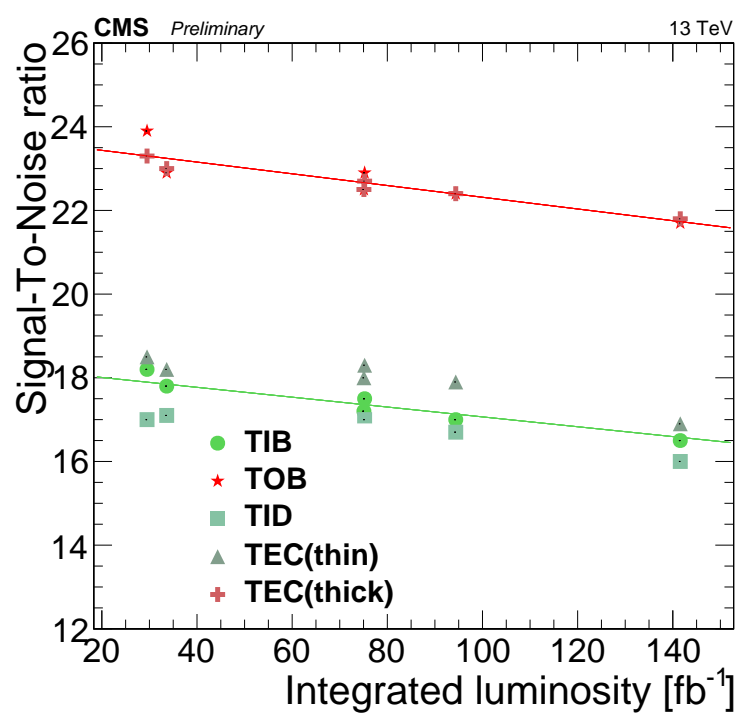

Figure 4: $\mathrm{S} / \mathrm{N}$ as a function of integrated luminosity.

The single hit efficiency is measured as a function of the instantaneous luminosity. Tracks passing so-called high purity criteria [6] of the tracking algorithm are used for the measurement. If the trajectory starts or ends with a considered module, it is discarded for computing the efficiency of that module. Thus, measurement in the first layer, TIB layer 1 is relying on pixel seeding and moreover no measurement is possible in the outermost regions, i.e. TOB layer 6 and TEC disk 9. The hit efficiency scales linearly with the instantaneous luminosity and pileup, as can be seen in Fig. 5. It is greater than 98\% and depends on the layer of the detector. Layers are affected differently because of the different probability of Highly Ionizing Particles (HIP) to pass the silicon sensors ${ }^{1}$. The average HIP event occurrence per p-p interaction per unit volume is shown in Fig. 6.

\footnotetext{
${ }^{1}$ HIPs are particles that can deposit the energy equivalent of $\sim 100$ Minimum Ionizing Particles (MIP).
} 
It can be seen that the HIP probability decreases with radius which comes from the inverse-square law of particle fluence.

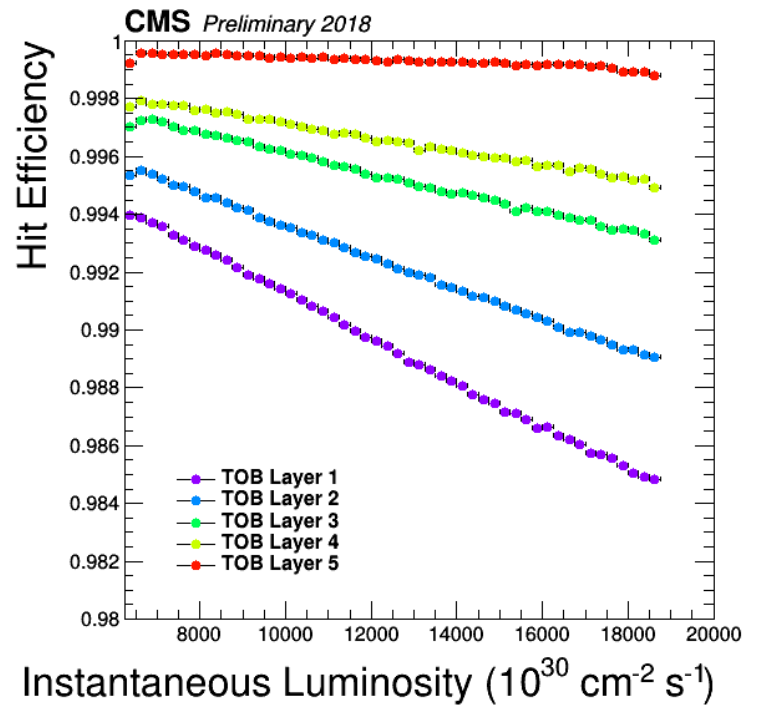

Figure 5: Single hit efficiency of silicon strip modules from 5 TOB layers as a function of the instantaneous luminosity[5].

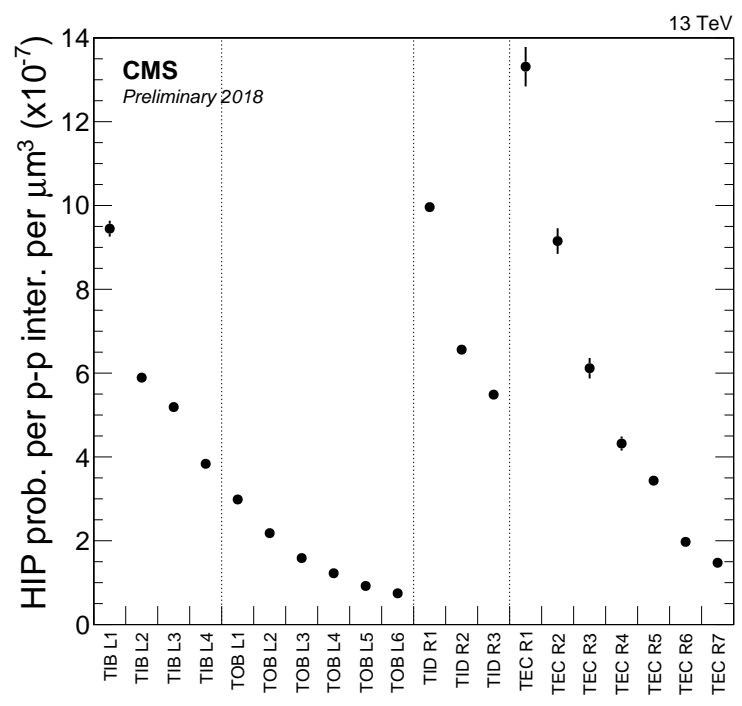

Figure 6: Average probability of HIP event occurrence per p-p interaction per unit volume for all layers of the Silicon Strip Tracker. In the TID and TEC, the probability is reported per ring[5].

An estimation of the hit resolution is performed with the so-called pair method based on tracks passing the detector through overlapping modules from the same tracking layer. Tracks with $p_{T}>3 \mathrm{GeV} / \mathrm{c}$, at least 6 hits in the strip tracker and $\chi^{2}$ track fit probability $>10^{-3}$ are selected. 
Clusters of the same size are considered. The resolution is computed as:

$$
\sigma_{\text {hit }}=\frac{\sqrt{\sigma_{\text {meas }- \text { pred }}^{2}-\sigma_{\text {pred }}^{2}}}{\sqrt{2}}
$$

where $\sigma_{\text {pred }}$ is extracted from the fit of difference in predicted hit positions to normal distribution, and $\sigma_{\text {meas-pred }}$ is extracted from the fit of double difference of measured and predicted hit positions to the normal distribution [6]. The measured hit resolution is shown in Fig.7 where resolution of binary readout is shown for comparison demonstrating that the hit resolution of the SST benefits from charge sharing due to analog readout.

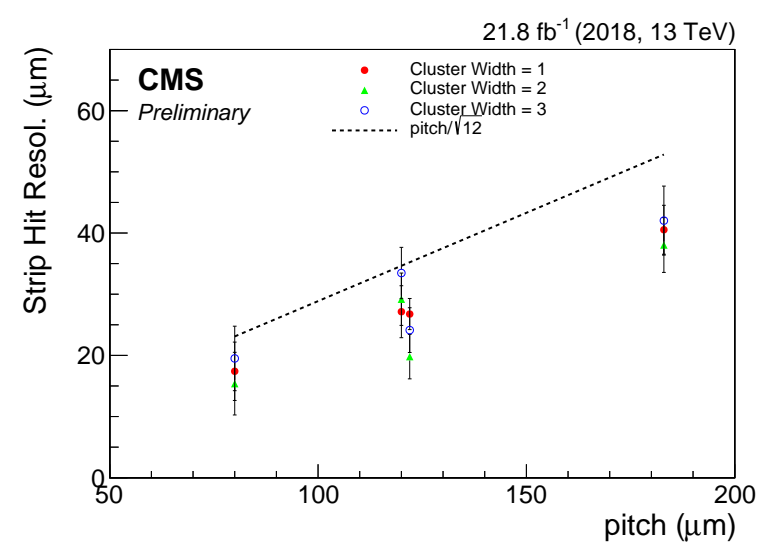

Figure 7: Strip hit resolution derived with the pair method by selecting pairs of hits in different types of overlapping sensors and for different cluster widths expressed in units of number of strips. The expected resolution for the binary readout (pitch $/ \sqrt{12}$ ) is also shown for comparison, demonstrating the benefit from charge sharing due to the analog readout [5].

The effect of interstrip capacitance is measured (cross-talk): the signal collected in a single strip induces charge on neighboring strips. If the total collected charge is $Q$, the leading strip receives a contribution $x_{0} \cdot Q$, the next two closest strips receive $x_{1} \cdot Q$, and the next ones $x_{2} \cdot Q$, so that:

$$
x_{0}+2 \cdot x_{1}+2 \cdot x_{2}=1
$$

The measurement is done with a dataset of cosmic muons taken when the CMS magnet is at 0 Tesla with data without zero-suppression [2]. Tracks from muons that pass the whole tracker with the trajectory almost normal to the detector planes are selected. Hits from the bottom part of the detector are selected with a trajectory in the same direction as in pp collisions. The measured crosstalk parameters are shown in Fig. 8. As can be seen in the figure, the clusters are narrower in the 2018 data than in the data measured in 2010. The new measurements have been introduced in the simulation software and have improved the cluster width description [5]. 


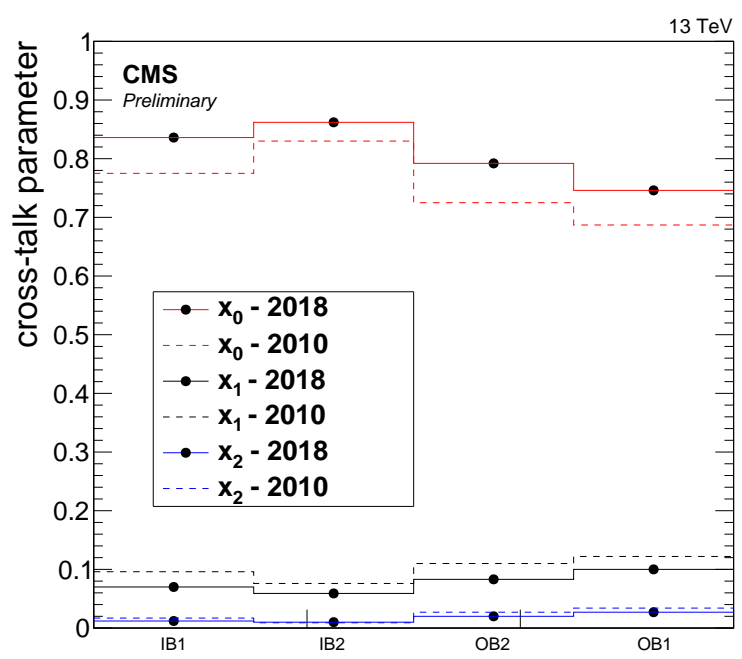

Figure 8: Cross-talk parameters for the four barrel silicon strip sensor geometries measured in 2018 (black dots) and prior to the data-taking (hatched lines) [5]. IB1 indicates type of modules used in the TIB layers 1 and 2, IB2 in the TIB layers 3 and 4, OB2 in the TOB layers 1-4, OB1 in the TOB layers 5 and 6 [3].

\section{Radiation effects}

By the end of 2018 the CMS Strip Tracker received a radiation dose corresponding to integrated luminosity of $200 \mathrm{fb}^{-1}$. Radiation effects are continuously monitored and they include: increase in leakage current, change of full depletion voltage due to the change of effective sensor doping concentration and evolution of laser driver performance in the optical readout chain.

Leakage current is measured both with Detector Control Units (DCU) which are ASICs located on each module and power supply unit [3]. In 2017 some of the HV channels (mostly in the first layer of TIB) were getting close to the power supply current limit (12 mA). The affected modules are located in the regions with degraded cooling contacts or with closed cooling loops (passive cooling). Thermal runaway was observed for several modules in the first layer of TIB and is shown in Fig.9. Modules that experience thermal runaway are stereo modules, where each side is powered by a different HV channel. As can be seen from Fig. 9, when one HV channel switches off because it reached the current limit, the self heating effect is greatly reduced for the other half of the modules. Reducing the bias voltage to $200 \mathrm{~V}$ significantly reduced the leakage current and allowed to operate these modules.

By the end of 2017 it was clear that there was no margin for regions of the detector with passive cooling to operate for all of 2018 with cooling at $-15^{\circ} \mathrm{C}$ without reaching the power supply current limit. In the beginning of 2018 the operational temperature of the detector cooling was changed to $-20^{\circ} \mathrm{C}$ and the detector was recalibrated before the start of data-taking. 


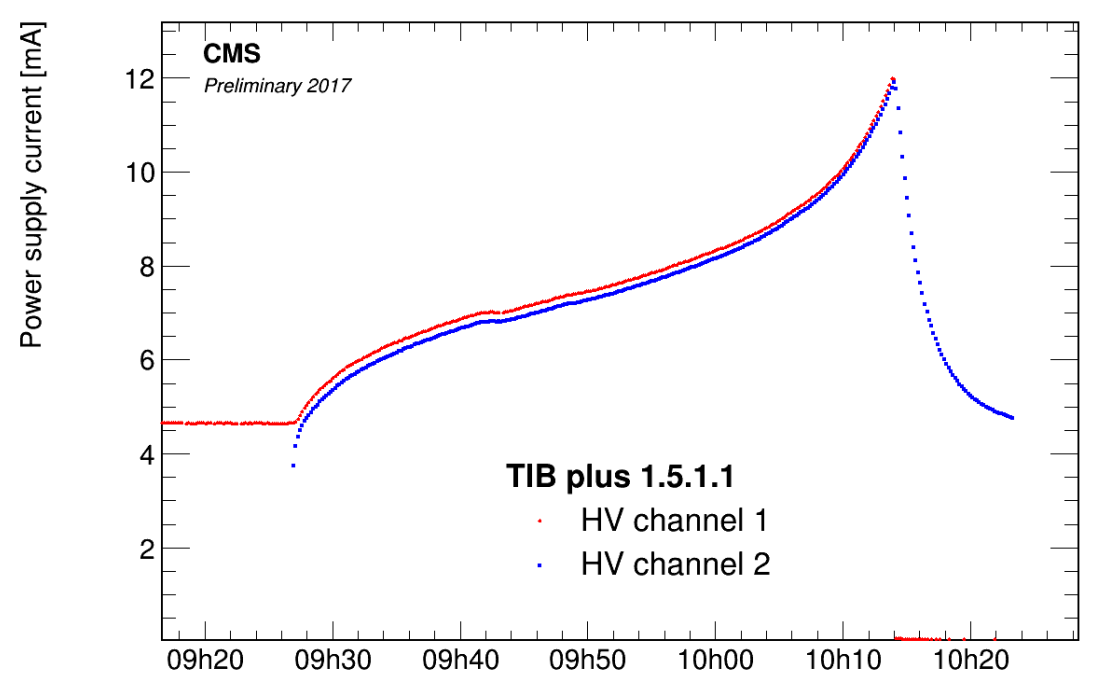

Figure 9: Thermal runaway of one power group in the first layer of TIB .

The full depletion voltage is measured during bias scans of the full detector (taken twice per year) and bias scans of selected modules (taken once per month on selected modules). Cluster width is used for measurement of the full depletion voltage. The method is illustrated in Fig.10(a): the ascending and plateau regimes of the curve are fitted with a linear function and their crossing point is taken as a measurement of the full depletion voltage. It can be noticed that plateau regime is not exactly flat: this can be explained by timing effects which are more pronounced at low bias voltages. The uncertainty of the method increases when the depletion voltage is close to the inversion point. As can be seen in Fig.10(b), curves taken during bias scans get shifted towards lower bias voltage indicating that the full depletion voltage decreases with integrated luminosity. Bias scan results have been compared with $\mathrm{C}-\mathrm{V}$ measurements done during production phase of the CMS tracker and two approaches were found to be in agreement [9].

The decrease of the full depletion voltage for each layer (ring) is shown in Fig.11 at an integrated luminosity of about $100 \mathrm{fb}^{-1}$ taking the initial depletion voltage into account. It can be expected that the first layers of TIB and TOB should be below $100 \mathrm{~V}$ and at an integrated luminosity of about $200 \mathrm{fb}^{-1}$ they are around the inversion point (where the method described is not very reliable). 


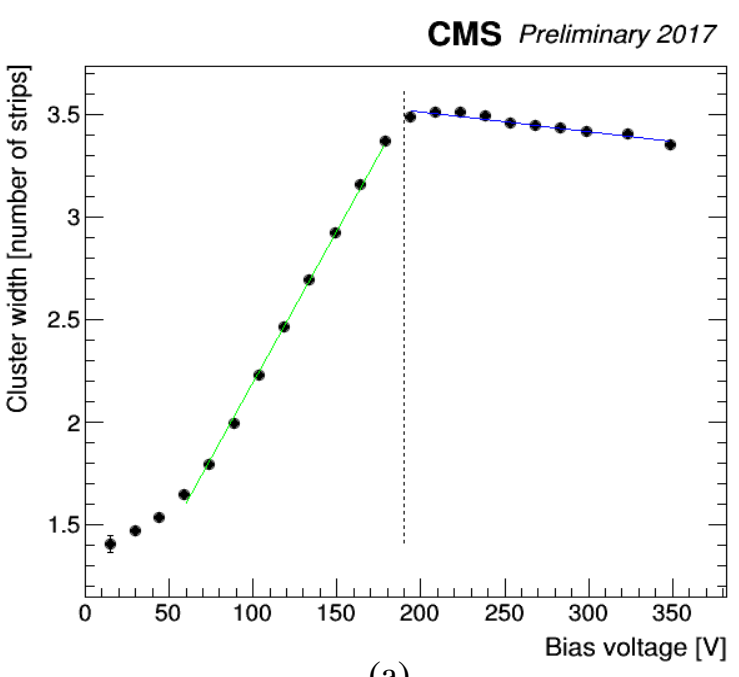

(a)

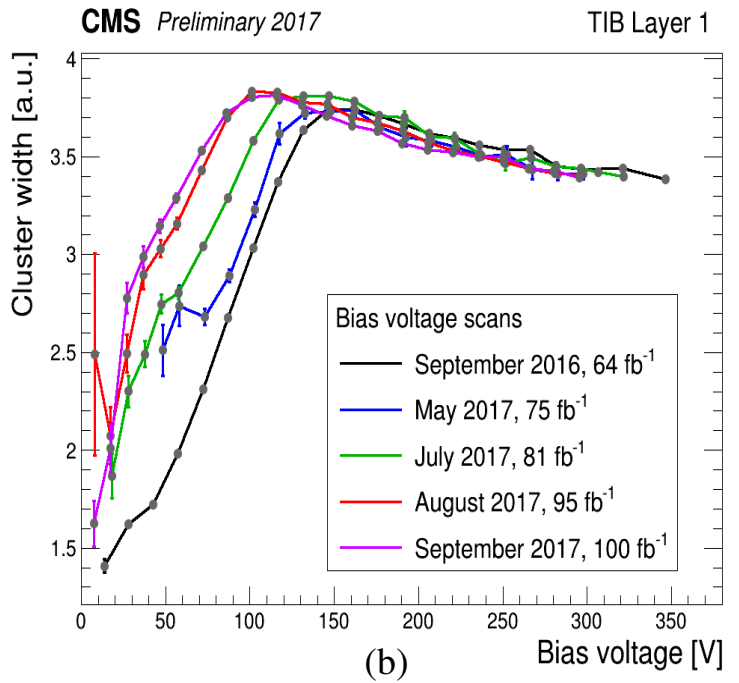

(b)

Figure 10: (a) Illustration of the method used to measure the full depletion voltage. (b) Cluster width (in arbitrary units) versus bias voltage for one module in the first layer of TIB.

\section{CMS Preliminary}

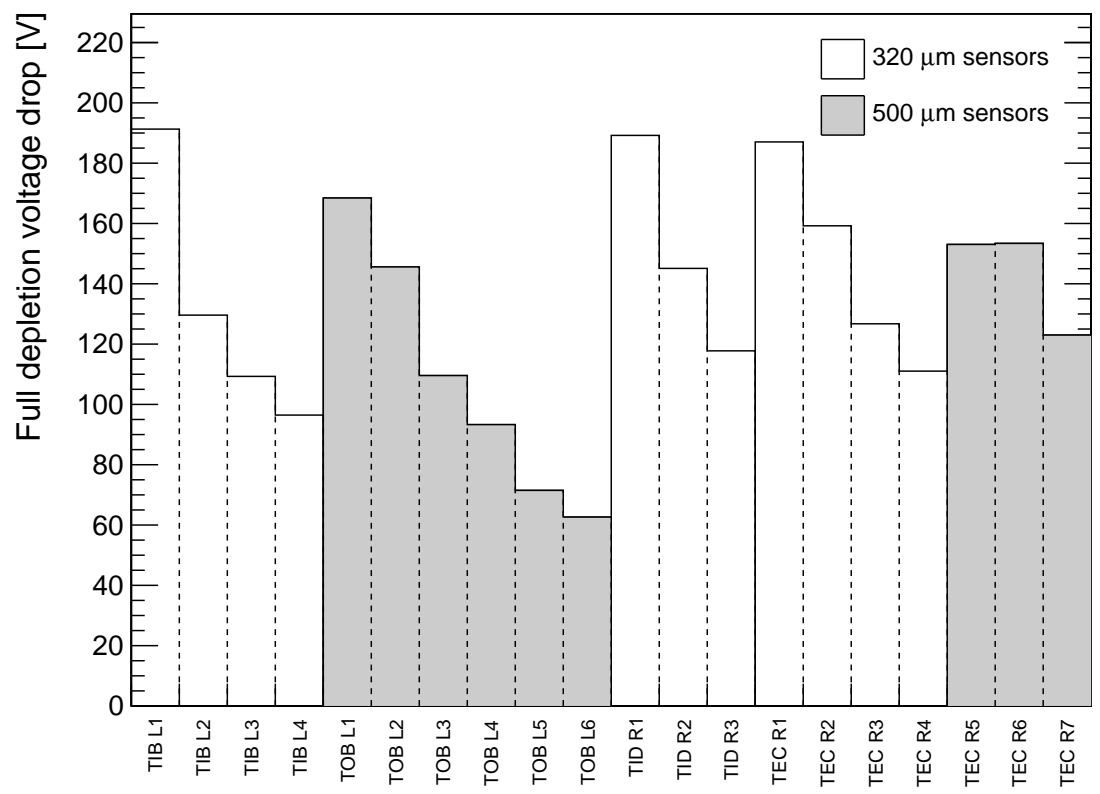

Figure 11: Decrease of the full depletion voltage for each layer computed as the difference between the values measured at the time of the CMS Tracker construction [9] and the values obtained by the analysis of the bias voltage scan of September 2017 involving all tracker modules.

The expected full depletion voltages of the strip detector modules are simulated using the Hamburg model [8] for a realistic future scenario after an integrated luminosity of $400 \mathrm{fb}^{-1}$. In Fig. 12 Tracker maps ( $z-\phi$ representation of the strips tracker layers and $x-y$ for rings) where modules that are expected to experience a thermal runaway with coolant temperature at $-20^{\circ} \mathrm{C}$ and $-25^{\circ} \mathrm{C}$ are highlighted. As it can been seen from the figure, the number of modules with thermal 
runaway can be decreased roughly by a factor of 2 by decreasing the coolant temperature to $-25^{\circ} \mathrm{C}$.

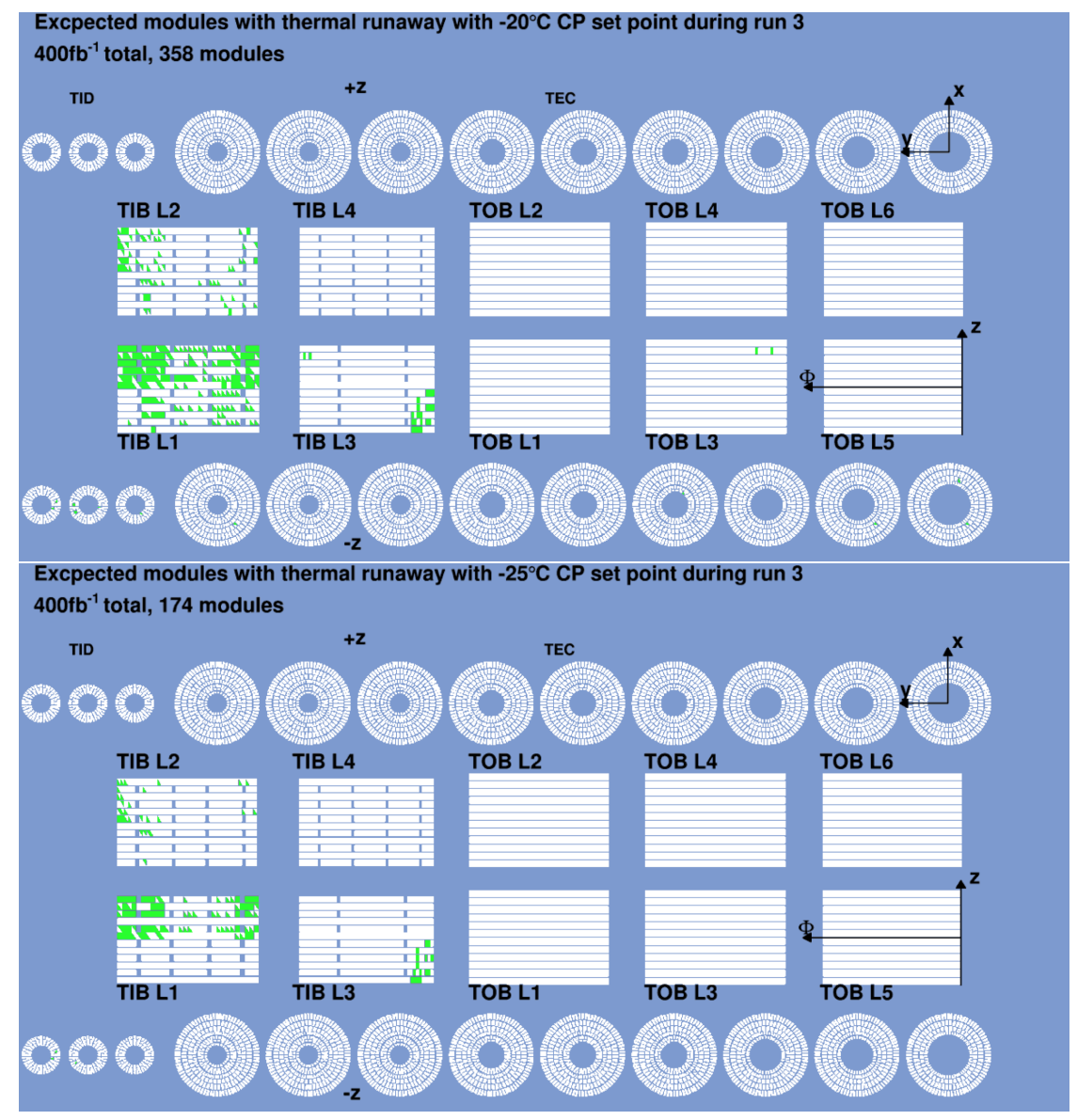

Figure 12: Expected modules with thermal runaway at integrated luminosity of $400 \mathrm{fb}^{-1}$ with coolant temperature of $-20^{\circ} \mathrm{C}$ (top) and $-25^{\circ} \mathrm{C}$ (bottom).

Radiation effects in the optical readout include increase of the laser driver threshold (minimum current needed to get non-zero signal from the laser diode) and loss of efficiency of a laser. The evolution of the laser driver current threshold increase is shown in Fig. 13 for different layers of the TIB. As can be seen in Fig. 13, the current laser driver threshold increases with irradiation during data-taking and anneals during end of year technical stops. The threshold current also decreases when the operational temperature is changed from $-15^{\circ} \mathrm{C}$ to $-20^{\circ} \mathrm{C}$. The average current at the time of reference was about $3 \mathrm{~mA}$ and the maximum allowed current is $22.5 \mathrm{~mA}$, which indicates that the optical system of the tracker strips has quite some margin for operation. 


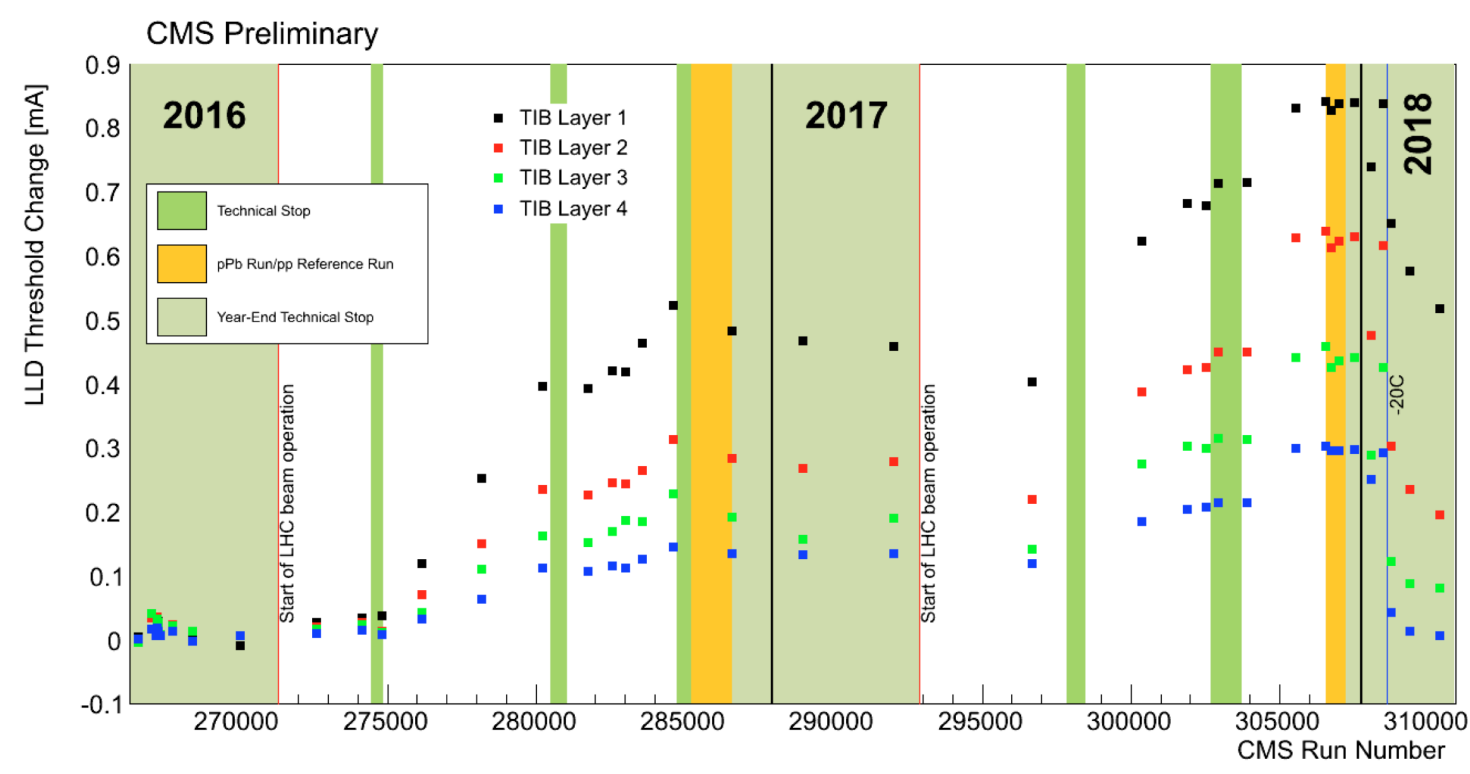

Figure 13: Evolution of the laser driver current threshold in the TIB versus runs taken by the CMS expertiment. The CMS run number is associated with a data-taking period and increases with time.

\section{Summary and outlook}

To summarize, it can be said that the CMS Silicon Strip Tracker is performing well by the end of 2018 after ten years of operation. Together with the other subsystems of the CMS experiment it continues to deliver high quality data for physics analyses. The system has not shown any significant degradation over the last years. Signal to noise ratio, hit efficiency and hit resolution are very good and compatible with the expectations. The operational temperature of the detector has been changed from $-15^{\circ} \mathrm{C}$ to $-20^{\circ} \mathrm{C}$, which helped to decrease the leakage current in regions with degraded cooling. The detector was recalibrated and commissioned at the new operational temperature at the beginning of 2018. Radiation effects are monitored continuously and are visible in all parts of the detector. The first layers of TIB and TOB are around the space charge inversion point at the end of Run 2. Regions of the detector with passive cooling should benefit from leakage current annealing during Long Shutdown 2 (LS2) when the detector will be kept cold (except approximately 120 days due to the extraction of the pixel detector and beam pipe extraction when the detector will stay at room temperature). Simulation studies suggest that the number of thermal runaways can be reduced by a factor of two when changing the operational temperature to $-25^{\circ} \mathrm{C}$. The test of running the detector at this temperature is foreseen to take place during LS2.

\section{References}

[1] F. Hartman, Evolution of Silicon Sensor Technology in Particle Physics, Springer International Publishing AG, 2017,DOI 10.1007/ 978-3-540-44774-0.

[2] O. Pooth, The CMS Silicon Strip Tracker Concept, Production, and Commissioning, Springer-Verlag New York Incorporated, 2010. 
[3] CMS Collaboration, "The CMS experiment at the CERN LHC" JINST 3 (2008) S08004.

[4] M. J. French et al.,"Design and results from the APV25, a deep sub-micron CMOS front-end chip for the CMS tracker" Nucl. Instrum. Meth. A 466, 359 (2001)

[5] CMS Collaboration, "Silicon Strip Tracker Performance results 2018", CMS-DP-2018-052, https://cds.cern.ch/record/2638062, 2018.

[6] CMS Collaboration, "Description and performance of track and primary-vertex reconstruction with the CMS tracker" JINST 9 (2014) no.10, P10009.

[7] W. de Boer et al., "The performance of irradiated CMS silicon micro-strip detector modules", CMS-NOTE-2006-049, http://cdsweb.cern.ch/record/951391.

[8] M. Moll, Radiation "Damage in Silicon Particle Detectors", Universität Hamburg, DESY-THESIS-1999-040, 1999, https://mmoll.web.cern.ch/mmoll/thesis/.

[9] Ch. Barth, "Performance of the CMS Tracker under Irradiation", KIT, Karlsruhe, Dept. Phys., 2013, http://cds.cern.ch/record/1972454. 